\title{
Cerebral potentials evoked by oesophageal distension in patients with non-cardiac chest pain
}

\author{
A J P M Smout, M S DeVore, C B Dalton, D O Castell
}

\begin{abstract}
Cerebral evoked potential recording was used to study the oesophagus-brain axis in $\mathbf{1 0}$ controls and 10 patients with non-cardiac chest pain with a manometric diagnosis of either nutcracker oesophagus or diffuse spasm and a positive edrophonium test. A series of $\mathbf{5 0}$ inflations (10/minute; inflation rate of $170 \mathrm{ml} /$ second) of an intraoesophageal balloon $(5 \mathrm{~cm}$ proximal to the lower oesophageal sphincter) was performed in each subject. Three different inflation volumes were used and were individually determined to cause no sensation, slight sensation, and definite sensation, respectively (volume ranges: $2-8 \mathrm{ml}, 5-18 \mathrm{ml}$, and 8-22 $\mathrm{ml}$ ). All signals were coded and their quality was scored on a scale from 0 (no recognisable pattern) to 5 (well defined potential of good quality) by four 'blinded' observers. The evoked potential quality scores and amplitude of the major peaks increased significantly $(\mathbf{p}<0.01)$ with increasing sensation, both in patients and in controls. In the patients, quality score and amplitude of all four peaks of the evoked potentials were lower $(p<0.05)$ and latencies of two of the four peaks were longer $(p<0.02)$ than in the controls. The volumes of air required to produce the various sensations were lower in the patients $(p<0.01)$. When divided by the balloon volume, amplitude and quality of the evoked potential were no longer significantly different between the groups. These results suggest that the increased perception of oesophageal distension in patients with non-cardiac chest pain is caused by altered central processing rather than (functionally) abnormal receptors in the oesophageal wall.
\end{abstract}

Gastroenterology,

Bowman Gray School of

Medicine, Winston-

Salem, North Carolina,

USA

M S DeVore

C B Dalton

Division of

Gastroenterology,

Jefferson Medical

College, Philadelphia,

Pennsylvania, USA

D O Castell

Correspondence to:

Dr A J P M Smout,

Department of

Gastroenterology, University Hospital, PO Box 85500, 3508 GA Utrecht, The Netherlands.

Accepted for publication

10 June 1991 The non-cardiac chest pain syndrome is characterised by recurrent angina like pain that cannot be explained by any abnormalities found at detailed cardiological investigations. Besides gastro-oesophageal reflux and oesophageal motor disorders, a lowered pain threshold for oesophageal stimuli has been suggested as a pathophysiological factor in this syndrome. ' One of the manifestations of the lowered oesophageal pain threshold in patients with non-cardiac chest pain is an increased sensitivity to balloon distension of the oesophagus. ${ }^{2}$ It is not known whether this is caused by a decreased threshold of receptors in the oesophageal wall, abnormal impulse conduction, or altered processing of information in the central nervous system.

Recently, electrical and mechanical stimulation of the oesophagus has been shown to evoke consistent cerebral potentials in man..$^{3-5}$ These cortical electrical potential variations probably reflect central processing of the sensory information that originates in the oesophagus. ${ }^{+}$

In this study we used cerebral evoked potential recording to study the transmission of sensory information from the oesophagus to the cerebral cortex in patients with non-cardiac chest pain. The study aimed to shed more light on the mechanism responsible for the abnormal perception of oesophageal balloon distension in this syndrome.

\section{Methods}

Experiments were carried out in 10 patients and 10 healthy subjects. The patients (six men and four women) ranged in age from 40 to 59 years (mean 47.5 years). All patients had had intermittent angina like chest pain for more than six months and had had negative cardiological investigations, which included exercise testing in all and coronary angiography in eight. In all patients a manometric diagnosis of either nutcracker oesophagus (seven patients) or diffuse oesophageal spasm (three patients) had been made. All had a positive edrophonium test - that is, their typical chest pain could be reproduced by iv injection of $80 \mu \mathrm{g} / \mathrm{kg}$ edrophonium chloride (Tensilon). None of the patients had undergone surgery of the oesophagus or stomach.

The 10 healthy subjects (seven men and three women) ranged in age from 23 to 60 years (mean 31.4 years). No volunteer had a history or neurological or gastrointestinal disease and none used drugs.

The experiments were carried out at the

Bowman Gray School of Medicine, WinstonSalem, North Carolina, USA. The study protocol was approved by the Human Research Review Committee of the Bowman Gray School of Medicine. All experiments were carried out in the early afternoon in subjects who had been fasting since breakfast.

A 9 lumen polivinyl catheter (OD $4.5 \mathrm{~mm}$, Wilson-Cook, Winston-Salem, North Carolina, USA) was introduced through the nose. This catheter carried a silicone rubber balloon (length of $30 \mathrm{~mm}$ ) connected to the central lumen of the catheter. The catheter was positioned with the 


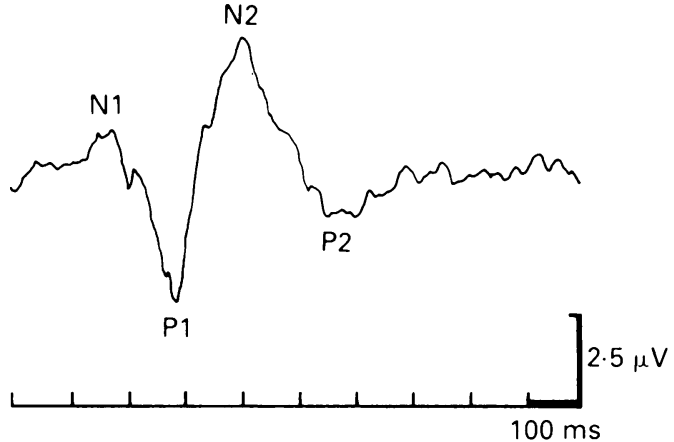

Figure 1: Configuration of cerebral evoked potentials recorded after oesophageal balloon distension. The well defined evoked potential shown in this figure, recorded at definite sensation volume in one of the healthy subjects, obtained a mean quality score of $4 \cdot 75$.

centre of the balloon at $5 \mathrm{~cm}$ proximal to the upper border of the lower oesophageal sphincter. Localisation of the lower oesophageal sphincter was made possible by intraluminal pressure recording through a side hole orifice $50 \mathrm{~mm}$ distal to the centre of the balloon.

Balloon inflation was accomplished by a specially designed pump powered by compressed air. This pump rapidly moved the plunger of a syringe, providing inflation and deflation at a speed of $170 \mathrm{ml} / \mathrm{second}$. The inflation volume was adjustable from 2 to $30 \mathrm{ml}$. The frequency of inflation was set at $10 /$ minute, the dwell time (time with balloon filled) was set at 1 second. The pump produced a $5 \mathrm{~V}$ logic pulse at the beginning of each inflation cycle, which was used to trigger the cerebral evoked potential recorder.

In each subject three volumes of balloon inflation were used, leading to no, slight, and definite sensation in the chest respectively. These three levels of inflation volume were individually determined before recording, using the pump described above. This was done as follows: three series of stepwise balloon distension (from $0 \mathrm{ml}$ upwards in $2 \mathrm{ml}$ increments, until $20 \mathrm{ml}$ or moderate or severe pain sensation) were administered in each subject. ${ }^{2}$ The subjects scored their sensation in the chest on a scale from 0 to 6 , as follows: 0 : no sensation, 1: slight sensation, 2: moderate sensation, 3: definite sensation, 4: slight pain, 5: moderate pain, 6: severe pain. In each subject, the mean of the three volumes at which score 1 was reached was taken as the 'slight sensation' volume. Likewise, the mean of the three

Control subject

No

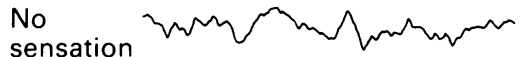

Slight
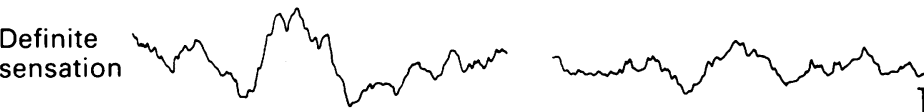

$2 \cdot 5 \mu \mathrm{V}$

$100 \mathrm{~ms}$

Figure 2: Typical series of evoked potentials recorded at no, slight, and definite sensation levels in a control subject and in a patient. Inflation volumes used were 5, 12, and $17 \mathrm{ml}$ in the healthy subject and 3, 9, and $13 \mathrm{ml}$ in the patient. volumes at which 'definite sensation' volume was reached was taken as 'definite sensation' volume. For the 'no sensation' volume, a volume $2 \mathrm{ml}$ lower than the lowest score 1 volume was taken.

Cerebral evoked potentials were recorded from a standard electroencephalographic electrode attached to the vertex (position $\mathrm{CZ}$ of the International 10-20 System). ${ }^{6}$ The reference electrode was placed at the right earlobe. Subjects were grounded with an electrode on the left earlobe. All electrodes were attached to the skin with collodion after careful cleaning and abrasion. The electrode resistance (for a $1 \mathrm{kHz}$ current) was below $5 \mathrm{k} \Omega$ in all cases. Signals were amplified, sampled $(1000 \mathrm{~Hz})$, and averaged with a Nicolet CA 1000 evoked potential averager. High and low pass filters ( $3 \mathrm{~dB} /$ octave) were set at 1 and $250 \mathrm{~Hz}$, respectively. Epochs of 1 second triggered by the onset of inflation were used. Fifty sweeps were averaged. On all records, upward deflections indicated negativity of the exploring electrode $(\mathrm{CZ})$ in respect of the reference electrode.

Throughout the cerebral evoked potential recording sessions the subjects were awake, lying supine, with eyes closed, in a dimly lit room. The sound of the pump was masked by administering white noise via in-ear headphones at a sound pressure level of $80 \mathrm{~dB}$. None of the subjects was able to hear the pump under these conditions. In all experiments a series of 'sham inflations' was included. In these, recordings were made with the pump running but with the balloon unattached. The pressure in the balloon was continuously monitored throughout the experiment. This was done using a T-piece on the nozzle, which was connected to a pressure transducer which in turn was connected to a Beckman physiograph.

\section{ANALYSIS}

At the completion of the experiments all recorded evoked potential signals were coded and independently evaluated during one reading session by four blinded observers. The analysis comprised both qualitative and quantitative assessment of the signals. In the qualitative assessment a score of 0 to 5 was attached (by each of the four observers) to each signal. These quality scores were defined as follows: 0 , no recognisable pattern; 1 , doubtful or uncertain whether evoked potential present; 2, pattern present; but evoked potential of poor quality; 3 , evoked potential of medium quality; 4 , evoked potential of good quality; and 5, very well defined evoked potential of good quality. The scores given by the four observers were averaged. Quantitative analysis was carried out when the average qualitative score for the evoked potential signal was 1 or higher. Latencies of the prominent peaks (positive and negative) were measured in milliseconds in respect of trigger onset. Amplitudes were measured in $\mu \mathrm{V}$ as the potential difference between consecutive peaks (positive to negative or negative to positive). All values are expressed as mean (SEM), unless stated otherwise. Two factorial analysis of variance (ANOVA) was used to investigate the difference between patients and healthy controls and the 


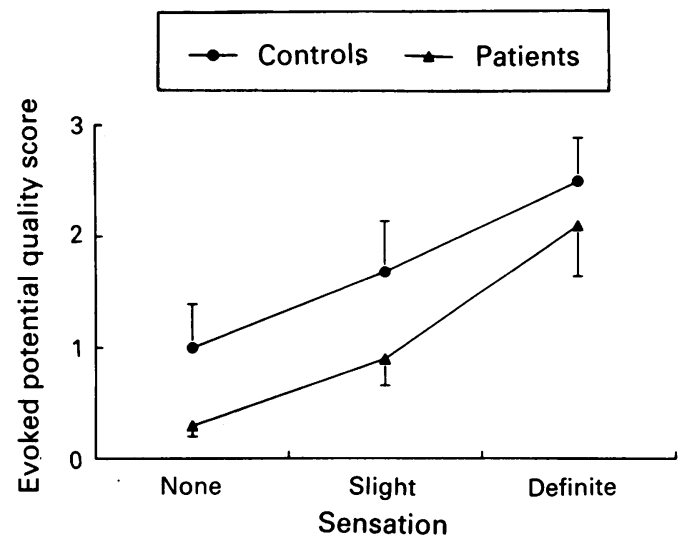

Figure 3: Evoked potential quality scores (mean (SEM)) in the 10 patients and the 10 healthy controls.

relation between chest sensation and the evoked potential characteristics.

\section{Results}

In none of the subjects did cerebral evoked potential recording with the balloon not attached to the pump ('sham inflation') result in a recognisable evoked potential pattern. The configuration of the recorded evoked potentials obtained after actual inflation was usually triphasic in both patients and controls. The four distinguishable
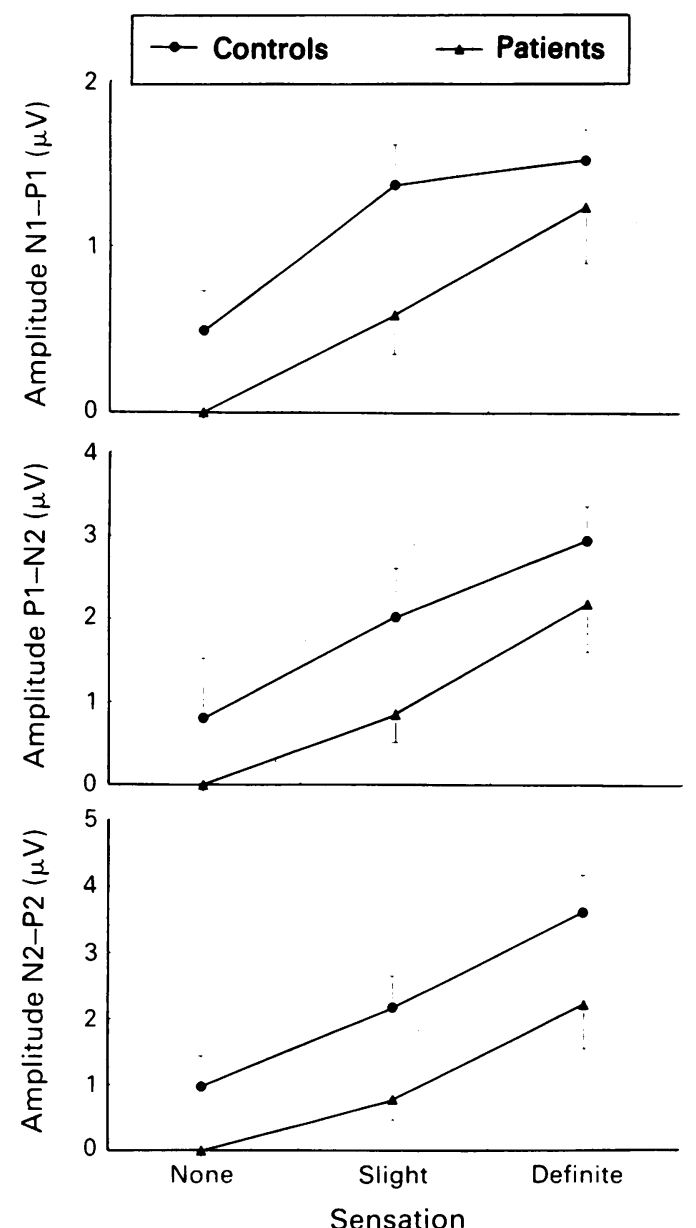

Figure 4: Evoked potential peak amplitudes (mean (SEM)) in the 10 patients and the 10 controls. Amplitudes of N1-PI, PIN2, and N2-P2 are shown in upper, middle, and lower panels, respectively.
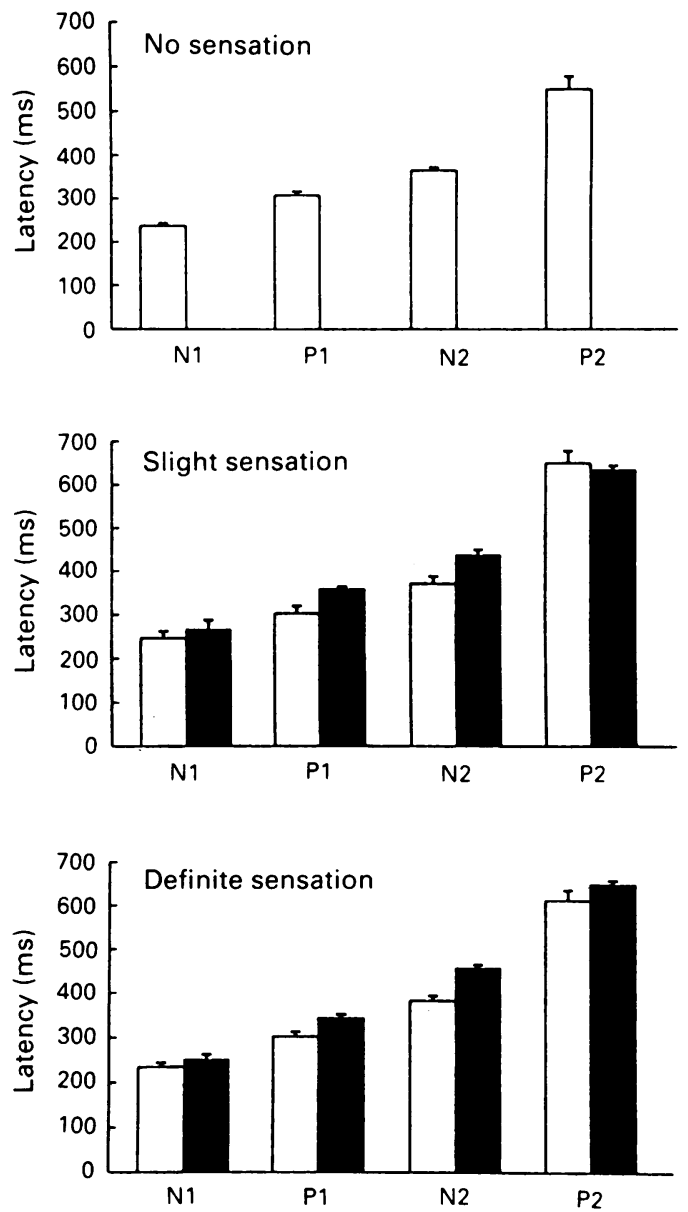

Figure 5: Latencies of cerebral evoked potential peaks (mean (SEM)) at no, slight, and definite sensation. Open bars= controls: closed bars = patients.

peaks were designated $\mathrm{N} 1, \mathrm{P} 1, \mathrm{~N} 2$, and $\mathrm{P} 2$ (Fig 1). Although no differences in waveform between patients and controls were observed, the patients typically had lower evoked potential amplitudes (Fig 2). With the lowest volume (not leading to perception of distension), only three of the healthy subjects and none of the patients had a readable evoked potential (score $\geq 1$ ). At the highest volume of inflation (leading to definite sensation) all healthy controls and six of the patients had a readable evoked potential. As shown in Figure 3, evoked potential quality scores were significantly lower in the patients than in the control subjects ( $p<0.05$, ANOVA) and quality scores increased significantly with increasing sensation, both in controls and in patients $(p<0 \cdot 01)$. Likewise, all evoked potential peak amplitudes increased significantly with increasing sensation $(\mathrm{p}<0.01)$ and peak amplitudes were lower $(p<0.05)$ in the patients than in the control subjects (Fig 4).

The latencies of the major peaks showed no significant correlation with chest sensation. At definite sensation, the latencies of $\mathrm{P} 1$ and $\mathrm{N} 2$ were significantly longer $(p<0.02$, Wilcoxon test) in the patients than in the controls (Fig 5).

The volumes of air required to produce the various sensation levels were lower $(p<0.02)$ in the non-cardiac chest pain patients than in the control subjects (Fig 6A). Accordingly, intraballoon pressures tended to be lower in the patients than in the controls, but the difference 

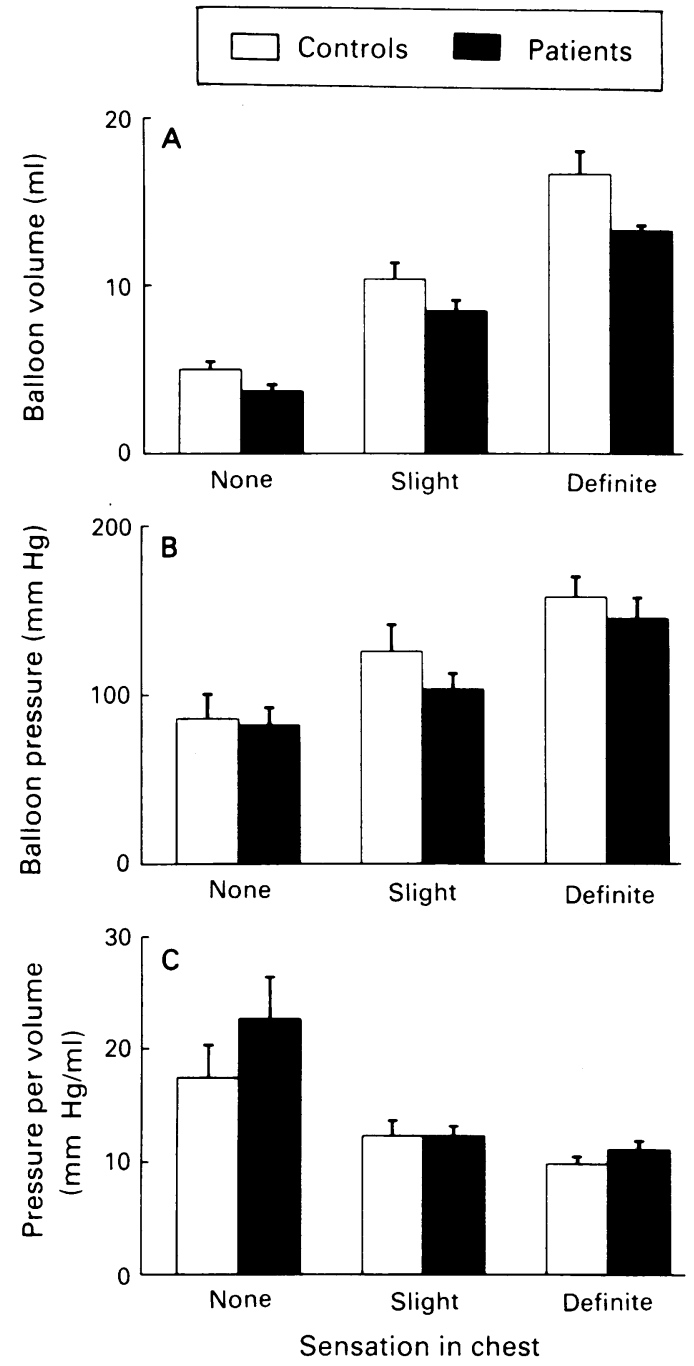

Figure 6: Volume and pressure in the intra-oesophageal balloon used to evoke cerebral potentials at no, slight, and definite sensation. Mean (SEM) of observations in 10 noncardiac chest pain patients and 10 healthy controls are shown. $A=$ balloon volumes; $B=$ intraballoon pressures; $C=$ pressure to volume ratios.

failed to reach statistical significance (Fig 6B). Pressure to volume ratios were not significantly different between the two groups (Fig 6C).

Evoked potential quality scores and amplitudes divided by the volume of inflated air were not significantly different between patients and controls (Table).

Evoked potential peak amplitudes and quality scores divided by the balloon volume at the three sensation levels in 10 control subjects and 10 patients with non-cardiac chest pain (values mean $(S E M))$

\begin{tabular}{|c|c|c|c|}
\hline & \multicolumn{3}{|c|}{ Sensation } \\
\hline & None & Slight & Definite \\
\hline \multicolumn{4}{|c|}{$\begin{array}{l}\text { Amplitude/volume }(\mu \mathrm{V} / \mathrm{ml}) \\
\text { Peak. }\end{array}$} \\
\hline N1-P1 Controls & $0.091(0.044)$ & $0 \cdot 145(0.054)$ & $0.094(0.013)$ \\
\hline Patients & $0.000(0.000)$ & $0.073(0.033)$ & $0.115(0.036)$ \\
\hline P1-N2 Controls & $0.153(0.075)$ & $0.209(0.071)$ & $0.196(0.039)$ \\
\hline $\begin{array}{l}\text { Patients } \\
\text { Pat }\end{array}$ & $0.000(0.000)$ & $0.103(0.043)$ & $0.197(0.058)$ \\
\hline N2-P2 Controls & $0.185(0.091)$ & $0.223(0.081)$ & $0.243(0.054)$ \\
\hline \multirow{2}{*}{\multicolumn{4}{|c|}{ Ouality score/volume }} \\
\hline & & & \\
\hline Controls & $0.205(0.077)$ & $0.169(0.052)$ & $0.165(0.033)$ \\
\hline Patients & $0.099(0.040)$ & $0.109(0.027)$ & $0.187(0.049)$ \\
\hline
\end{tabular}

No significant differences between patients and controls or between sensation levels were found.

\section{Discussion}

The genesis of the pain in the non-cardiac chest pain syndrome is still unclear. Even in the sharply defined group of patients studied by us, all of whom had a manometric diagnosis of either nutcracker oesophagus or diffuse oesophageal spasm and reproduction of the pain on edrophonium, the mechanism of pain generation has not yet been elucidated. In order to obtain more information about the abnormal pain perception in this syndrome we used the evoked potential technique. Evoked potentials are defined as the electrical responses of neuronal structures (nerves, spinal cord, cerebral cortex) to stimuli applied to sensory organs. In clinical neurophysiology, evoked potential recording has found applications in the assessment of visual perception, ${ }^{7}$ of auditory perception, ${ }^{8}$ and of somatosensory perception. ${ }^{9}$ Viscerosensory evoked potential recording after stimulation of the gastrointestinal tract has only recently been explored. ${ }^{1011}$ It has been shown that cerebral evoked potentials can also be recorded after stimulation of the oesophagus. ${ }^{3-5} \mathrm{We}$ wished to explore the possibility that evoked potential recording could be of help in explaining the pathogenesis of the non-cardiac chest pain syndrome. Although technically more demanding than electrical stimulation, we preferred to use balloon distension as a stimulus. Electrical stimulation is clearly less physiological than distension and may also stimulate neuronal structures other than receptors in the oesophageal wall. ${ }^{12}$

In the analysis of cerebral evoked potentials, emphasis is usually placed on measurement of peak latency - that is, the duration of the interval between stimulus and electrical response. Few attempts seem to have been made to quantitate the amplitude of the evoked potential. Since unblinded analysis of the evoked potential signals is susceptible to observer bias, we used an approach in which a panel of blinded observers interpreted all evoked potentials. In this analysis a quality score was used, since individual evoked potential peaks may be indistinguishable from background noise when a weak stimulus is used. The validity of such an approach was proved in a recent study in which a stimulus-response relation was found in normal subjects. ${ }^{5}$

Theoretically, the site of the abnormality in non-cardiac chest pain patients may be in one of the following three levels. Firstly, the abnormal oesophageal contractions that occur in many of these patients might, in themselves, cause the pain, for example by causing ischaemia of the oesophagus or be excessively stimulating stretch receptors in the oesophageal wall. The presence of abnormal oesophageal motility does not explain, however, why these patients have an increased sensitivity to passive oesophageal distension, as described by Richter et $a l^{2}$ and confirmed in this study.

Secondly, the stretch receptors in the oesophageal wall might be abnormal, in the sense that they fire more action potentials upon mechanical stimulation than normal. The decreased oesophageal sensation threshold to balloon distension found in this and a previous study $^{2}$ is consistent with this hypothesis. 
However, if this were the prinicpal mechanism involved, one would expect that on oeosphageal distension, non-cardiac chest pain patients would produce evoked potentials with a higher amplitude than normal subjects. In contrast, evoked potentials, were less well defined and had lower amplitudes in patients with this syndrome. One might argue that differences in the characteristics of the oesophageal wall - for example higher resting tone in the patients - are responsible for the decreased sensation threshold. However, neither intraballoon pressures nor the pressure to volume ratios in our patients were different from those in the controls.

Thirdly, central processing of otherwise normal oesophageal information may be abnormal in non-cardiac chest pain. The results of this study suggest that this mechanism does play a role in the generation of the pain in this syndrome. Compared with healthy controls, the non-cardiac chest pain patients had significantly lower evoked potential amplitudes associated with similar sensations in the chest. However, the cerebral evoked potentials generated by the patients had normal amplitudes when related to the degree of oesophageal distension applied (balloon volume). In other words, per $\mathrm{ml}$ of air inflated into the balloon, the non-cardiac chest pain patients generated evoked potentials that were about as large as those generated by control subjects. Nevertheless, the patients reported stronger sensations (per distension volume) than did the controls. These observations indicate that normal information reaches the brain of non-cardiac chest pain patients and that the increased perception of oesophageal distension in these patients is centrally located.

Virtually nothing is known about the site in the brain at which the recorded potentials are generated. The possibility that the potentials are non-specific late arousal potentials cannot be excluded completely, but evidence against this hypothesis has been produced. ${ }^{4}$ It is most likely that the cerebral potentials recorded after oesophageal distension originate in the cortical area where sensory information from the oesophagus is received or processed.

In summary, the observations made in this study suggest that our group of patients with non-cardiac chest pain have altered central processing of normal oesophageal sensory information, rather than abnormal oesophageal (stretch) receptors. It is unlikely, however, that this is the only mechanism involved in the pathogenesis of non-cardiac chest pain. Even in the rather sharply delineated group of patients studied by us the results showed considerable interindividual variability. It is to be expected that even greater variability will be met when patients with other possible causes of their chest pain, such as gastro-oesophageal reflux and the irritable oesophagus syndrome, are studied. Whether cerebral evoked potential recording will eventually prove to be of clinical value in the investigation of patients with (suspected) noncardiac chest pain is a question that cannot yet be answered.

The expert assistance of Mrs P Greene and the valuable advice of Drs E V Spudis, L M A Akkermans, and H Franssen are gratefully acknowledged.

The pump used to inflate the balloons was designed and constructed by the Department of Instrumentation of the University Hospital, Utrecht, The Netherlands. Dr Smout received a travel grant from the Netherlands Organisation for Scientific Research (NWO)

This work was presented in part at the meeting of the American Gastroenterological Association, San Antonio, Texas, USA, May 1990 , and published in abstract form in Gastroenterology 1990; 98: A393.

1 Vantrappen G, Janssen J, Ghillebert G. The irritable oesophagus: a frequent cause of angina-like pain. Lancet 1987; i: $1232-4$

2 Richter JE, Barish CF, Castell DO. Abnormal sensory perception in patients with esophageal chest pain. Gastroenterology 1986; 91: 845-52

3 Frieling T, Enck P, Wienbeck M. Cerebral responses evoked by electrical stimulation of the esophagus in normal subjects. Gastroenterology 1989; 97:475-8.

4 Castell DO, Wood JD, Frieling T, Wright FS, Vieth RF. Cerebral electrical potentials evoked by balloon distention of the human esophagus. Gastroenterology 1990; 98: 662-6.

5 Smout AJPM, DeVore MS, Castell DO. Cerebral potentials evoked by esophageal distension in humans. Am $\mathcal{f}$ Physiol evoked by esophageal

6 Jasper $\mathrm{HH}$. The $10-20$ electrode system of the international federation. Electroencephalogr Clin Neurophysiol 1958; 10: $371-5$

7 Stockard JJ, Hughes JF, Sharbrough FW. Visually evoked potentials to electronic pattern reversal: latency variations with gender, age and technical factors. Am $\mathcal{F} E E G$ Technol 1979; 19: 171-204.

8 Salamy A, McKean CM, Pettett G, Mendelson T. Auditory brainstem recovery processes from birth to adulthood. Psychophysiology 1970; 15:214-9.

9 Jones SJ. An interference approach to the study of somatosensory evoked potentials in man. Electroencephalogr Clin sensory evoked potentials in

10 Collet L, Meunier P, Duclaux R, Chery-Croze S, Falipou P. Cerebral evoked potentials after endorectal mechanica stimulation in humans. Am 7 Physiol 1988; 254: G477-82.

11 Frieling T, Enck P, Wienbeck M. Cerebral responses evoked by electrical stimulation of the rectosigmoid in normal subjects. Dig Dis Sci 1989; 34: 202-5.

12 Meunier PD. Endorectal cerebral evoked potentials (letter) Dig Dis Sci 1990; 35: 539. 